\title{
Gabor-HOG Features based Face Recognition Scheme
}

\author{
Hamid Ouanan*, Mohammed Ouanan, Brahim Aksasse \\ Department of Computer Sciences, Asia Team, Laboratory M2I, \\ Faculty of Sciences and Techniques, Moulay Ismail University, Morocco \\ *Corresponding author, email: ham.ouanan@gmail.com
}

\begin{abstract}
Extraction of invariant features is the core of Face RecognitionSystems (FRS). This work proposes a novel feature extractor-fusion scheme using two powerful feature descriptor known as Gabor Filters (GFs) and Histogram of Oriented Gradient (HOG), which the face image is filtered with the multiscale multiresolution Gabor filter bank to generate multiple Gabor magnitude images (GMIs), then the down-sampled GMIs and apply Histogram of Oriented Gradient to form the features. The experimental results on the FERET face database show the effectiveness of our methods.
\end{abstract}

Keywords: face recognition, gabor filters, histogram of oriented gradient, histogram matching

Copyright () 2015 Institute of Advanced Engineering and Science. All rights reserved.

\section{Introduction}

Because of the strong demand for public security in recent years, camera networks of intelligent surveillance have been distributed all over the world. Many new issues are raised [1, 2]. As one of the key technologies of intelligent surveillance, face recognition in surveillancehasattractedgrowinginterests [3-4].

Face recognition has become a popular area of research in computer vision and one of the most successful applications of image analysis and understanding [5]. It has received significant attention, especially during the past few years.Many methods of face recognition have been proposed during the past 30 years. Face recognition is such a challengingyet interesting problem that it has attracted researchers, neuroscientists and psychologists. It is due to this fact that theliterature on face recognition is vast and diverse [6]. A general statement of the problem of machine recognitionof faces can be formulated as follows: Given still image of a scene, identify or verify one or more persons in thescene using a stored database of faces [7].

Due to the nice mathematical property of Gabor function and its analogy to the biological mechanism, 2D Gabor filter has been widely used in face recognition. Gabor-based features have achieved excellent performance on the FERET database [8]. However, Gaborbased features all treated the multiple outputs of different Gabor filters separately and just stacked the features of each output together. The stacking procedure multiplies the feature dimension, leading to extremely high feature dimension, which imposes high computation and storage load.

In this paper, a novel Gabor-HOG feature extractor, which greatly reduces the feature dimension and retains the high performance, is proposed to overcome the problems caused byhigh feature dimension.Moreover, there is a biological motivation that orientation plays an important role in visual perception.

The paper is organized as follows. In Section 2, we briefly review some feature extractors, whose results are compared in this paper. The fusion alghoritmis presented in Section 3and the experimentsare explained in Section 4. Finally, Section 5 presentsconclusions.

\section{Feature Extractors}

This section briefly describes feature extractors used in thepresent work for face recognition studies. 


\subsection{Gabor Features}

The frequency and orientation representations of Gabor filters are similar to those of the human visual system and they have been found to be particularly appropriate for texture representation [9]. Gabor filters have been widely used in pattern analysis applications [9-10]. The most important advantage of Gabor filters is their invariance to illumination, rotation, scale, and translation. Furthermore, they can with stand photometric disturbances, such as illumination changes and image noise.

A 2D Gabor function $\mathrm{g}(\mathrm{x}, \mathrm{y})$ and its Fourier transform $\mathrm{G}(\mathrm{u}, \mathrm{v})$ are as follows:

$$
\begin{aligned}
& \mathrm{g}(x, y)=\frac{1}{2 \pi \sigma_{x} \sigma_{y}} \exp \left[-\frac{1}{2}\left(\frac{x^{2}}{\sigma_{x}^{2}}+\frac{y^{2}}{\sigma^{2} y}\right)+2 \pi j W x\right] \\
& \mathrm{G}(\mathrm{u}, \mathrm{v})=\exp \left[-\frac{1}{2}\left(\frac{(\mathrm{u}-\mathrm{W})^{2}}{\sigma_{\mathrm{u}}^{2}}+\frac{\vartheta^{2}}{\sigma_{\mathrm{v}}{ }^{2}}\right)\right]
\end{aligned}
$$

With $\rho_{\mathrm{u}}=1 / 2 \pi \rho_{\mathrm{x}}$ and $\rho_{\vartheta}=1 / 2 \pi \rho_{\mathrm{y}}$.

$$
g_{m n}(x, y)=a^{-m} G\left(x^{\prime}, y^{\prime}\right)
$$

Where, $\quad a>1 ; x^{\prime}=a^{-m}(x \cos \theta+y \sin \theta)$ and $y^{\prime}=a^{-m}(y \cos \theta-x \sin \theta), \quad \theta=n \pi / N$, for $m=$ $0,1, \ldots \mathrm{M}-1$ and $\mathrm{n}=0,1, \ldots \mathrm{N}-1, \mathrm{M}$ is the number of resolutions and $\mathrm{N}$ is the number of orientations.

The selection of parameters for the GF is a crucial issue. In the experiment, orientation (n) and scale $(\mathrm{m})$ as determining parametrs are selected using method introduced by Moreno et al [11]. Four orientations are used to capture the edge and texture and nine scales are used to capture the scale at wich the image is viewed. Using the above parametrs, we get a 36dimensional texture feature vector of each image.

\subsection{HOG Features}

Histogram of Oriented Gradients (HOG) [12] is inspired on Scale-Invariant Feature Transform (SIFT) descriptors proposed by [13]. To compose HOG, the cell histograms of each pixel within the cell casts a weighted vote, according to the gradient L2-norm, for an orientationbased histogram channel.

The HOG descriptor is similar to SIFT (Scale-invariantfeature transform) [12]. HOG descriptor is acquired throughthe following four steps. 1) Gradient calculation, 2) Histogramof gradient by cells, 3) Contrast normalize over overlappingspatial blocks, 4) Obtaining HOG descriptor. The first step tocompute HOG vectors is also similar to that presented forPOEM descriptors.

As described in the work $[14,15]$, the first step in extractingPOEM or HOG features is the computation of the gradientimage. The gradient orientation of each pixel is then evenlydiscretized over $0-\pi$ (unsigned representation) or $0-2 \pi$ (signed representation). Thus, at each pixel, the gradient is a2D vector with its original magnitude and its discretizeddirections.

The gradient consists of the convolution of two masksMaskx and Masky in each direction (for example [ $\left.\begin{array}{lll}-1 & 0 & 1\end{array}\right]$ et [ $\left.-\begin{array}{ll}-10 & 1\end{array}\right]$ ), that corresponds to $x$ and $y$ directions [15].

The gradient in pixel $(\mathrm{x}, \mathrm{y})$ in the image $I$ can be expressedas convolution of the masks with the original image:

$$
\begin{aligned}
& \mathrm{G}_{\mathrm{x}}(\mathrm{x}, \mathrm{y})=\operatorname{Mask}_{\mathrm{x}} * \mathrm{I}(\mathrm{x}, \mathrm{y}) \\
& G_{y}(x, y)=\text { Mask }_{y} * I(x, y)
\end{aligned}
$$

The magnitude of gradient in each pixel $(x, y)$ is calculated by the formula:

$$
\mathrm{G}(\mathrm{x}, \mathrm{y})=\sqrt{G(x, y)_{x}{ }^{2}+G(x, y)_{y}{ }^{2}}
$$

The corresponding direction is calculated by: 


$$
\varphi(x, y)=\arctan \left(\frac{G(x, y)_{y}}{G(x, y)_{x}}\right)
$$

As described in the majorty of works treating HOG,POEM parameters, the gradient orientation of each pixel isthen evenly discretized over $0-\pi$ (unsigned representation)or $0-2 \pi$ (signed representation) [15].

At each pixel, the gradient is a $2 \mathrm{D}$ vector with its originalmagnitude $\mathrm{G}(\mathrm{x}, \mathrm{y})$ and its discretized directions $\varphi(x, y)$. The second step consists of quantizing the gradientdirection in $\mathrm{m}$ orientations using the following equation:

$$
\theta(x, y)=\frac{\varphi(x, y)}{\left(\frac{\pi}{m}\right)} \operatorname{Or} \theta(x, y)=\frac{\varphi(x, y)}{\left(2 * \frac{\pi}{m}\right)}
$$

In this work the histogram channels are calculated over rectangular cells (i.e. R-HOG) by the computation of unsigned gradient. The cells overlap half of their area, meaning that each cell contributes more than once to the final feature vector. In order to account for changes in illumination and contrast, the gradient strengths were locally normalized, i.e. normalized over each cell. The HOG parameters were adopted after a set of experiments performed over the training data set. The higher Area Under Recall-precision Curve, computed over the validation data set, was achieved by means of 9 rectangular cells and 9 bin histogram per cell. The nine histograms with nine bins were then concatenated to make a 81 -dimensional feature vector.

\section{Fusion Algorithm}

In this section, we presente our proposed face-recognition system, which is composed of different parts as shown in Figure 1.

There are main steps in our proposed face representation with Gabor-HOG feature:

a) Filter the face image with the Gabor filter bank and calculate the magnitudes of the complex responses, aiming to obtain the Gabor magnitude images.

b) Compute Histogram of oriented gradient based on the Gabor magnitude images.

\subsection{Face Database}

Face databases are characterized by many criteria such as: facial expressions face zooming or scale (s), the time interval $(\mathrm{T})$ of the various catching sessions of photographs for the same individual, pose and illumination conditions.

Among the public databases available on the Internet, we have chosen to adopt the most popular one which are: Color FERET [16]. The FERET face image database is a result of the FERET program, which was sponsored by the US Department of Defense through the DARPA program. It has become a standard database for testing and evaluating state-of-the-art face recognition algorithms. Color FERET contains totally, 11338 pictures (face) that was obtained by photographing 994 subjects from different angles, during 15 sessions in the period from 1993 to 1996. SMALL sized directory images of FERET database is about $256 \times 384$ pixels and the files are PPM (Portable Pixel Map).Different Color FERET partitions are used in this work: $\mathrm{Fb}(1,195$ samples with expression variation), $\mathrm{Fc}(194$ samples with lighting variation), Dup.1(722 samples with 0-34 months of time interval), with $\mathrm{Fa}(1,196$ samples).

\subsection{Histogram Matching}

Since the Gabor-HOG feature is a sequence of histograms, the histogram intersection is applied as the similarity measure for histogram matching (HM). The histogramintersection is defined as:

$$
s\left(h_{1}, h_{2}\right)=\sum_{i=1}^{N} \min \left(h(i)_{1}, h(i)_{2}\right)
$$

Where $h_{1}$ and $h_{2}$ are two histograms, $h(i)_{1}$ and $h(i)_{2}$ are ith bin of $h_{1}$ and $h_{2}$ respectively, and $N$ is the number of histogram bins. 


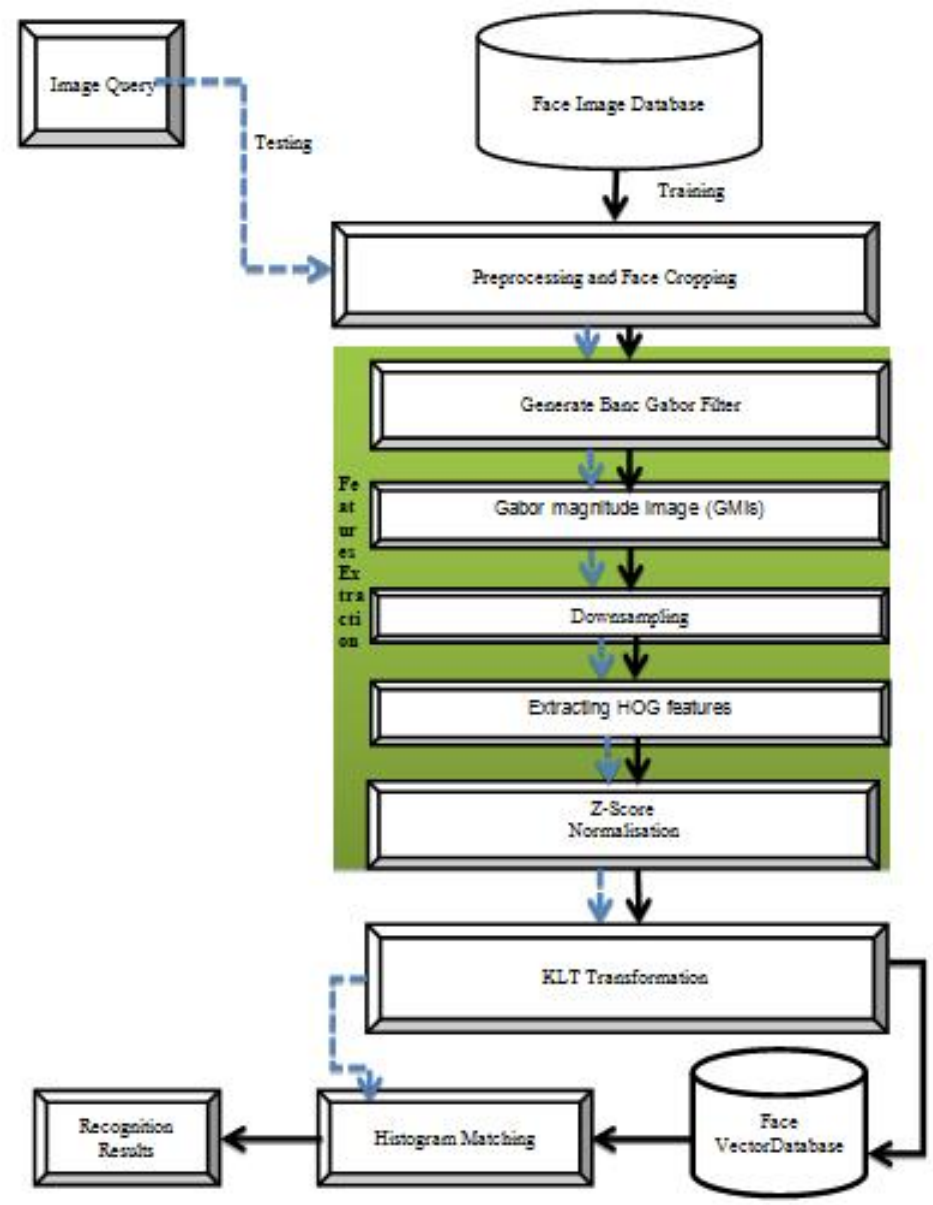

Figure 1. Illustration of the proposed approach for image based face recognition.

\section{Experiments}

A large scale experimental study has taken place in order to perform useful conclusions towards the recognition performance of Gabor-HOG feature extractor on the FERET database.

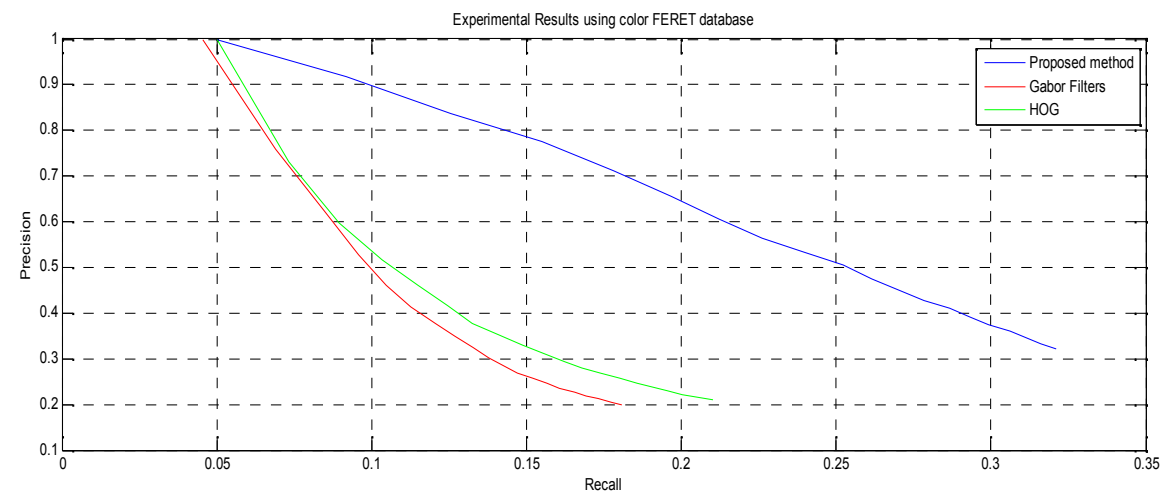

Figure 2. The Recall-precision curves of our proposed system compared to GFs and HOG Color FERET

From the above results, it can be observed that our proposed system outperforms over all existing face recognition system based on Gabor Filter, using the Color FERET database for 
reference. Best performances are observed by combining Gabor filters and Gradient of Oriented Histogram to form feature for face recognition.

\section{Conclusion}

In this paper we have presented in this paper a novel feature extractor (Gabor-HOG) gratlyreduces the feature dimension relative to otherGabor-based features while retains the high recognition performance. The procedure of our proposed feature exrator is very simple, which makes it very efficient to compute.

For future work, we propose to make comparison with other feature extractor like POEM and LBP, etc. other metric of similarity and classifier like SVM could be investigated to improve the recognition rate.

\section{References}

[1] Chunxiao Liu, Guijin Wang, Xinggang Lin. Multiple-shotper sonre identification by pairwise multiple instance learning. IEICE Transactionson Information and Systems 96. 2013; 12: 2900-2903.

[2] Liu Chunxiao, Wang Guijin, Lin Xinggang, LiLiang. Personre-identification by spatial pyramid color representation and local region matching. IEICE Trans-actions On Information And Systems 95. 2012; (8): 2154-2157.

[3] J Stallkamp, HK Ekenel, R Stiefelhagen. Video-based face recognitionon real-world data, In ICCV. 2007: 1-8

[4] Y Hu, AS Mian, R Owens. Sparse approximated nearest points for image set classification. In CVPR. 2011: 121-128.

[5] Kresimir delac, Mislav Grgic, Sonja Grgic. Independant comparative Study of PCA, ICA and LDA on the FERET data Set. Wiley periodicals, Inc. 2006; 15: 252-260.

[6] W Zhao, R Chellappa, P Phillips, A Rosenfeld. Face Recognition: A Literature Survey. ACM Computing Surveys. 2003; 35(4): 399-458.

[7] Kresimir Delac and Mislav Grgic. Editors. Wavelets and Face Recognition. Vienna, Austria: I-Tech. 2007: 558.

[8] W Zhao, R Chellappa, PJ Phillips. Subspace Linear Discriminant Analysis for Face Recognition. Center for Automation Research, University of Maryland, College Park, MD. 1999.

[9] Shen L, Bai L, Fairhurst M. Gabor wavelets and general discriminant analysis for face identification and verification. Image and Vision Computing. 2007; 25(5): 553-563.

[10] Liu C, Wechsler H. Gabor feature based classification using the enhanced fisher linear discriminant model for face recognition. IEEE Transactions on Image Processing. 2002; 11: 467-476.

[11] Plinio Moreno, Alexandre Bernardino, Jose Santos-Victor. Gabor Parameter Selection for Local Feature Detection. IbPRIA. 2005; (1): 11-19.

[12] Dalal N, Triggs B. Histograms of oriented gradients for human detection. In IEEE Computer Society Conference on Computer Vision and Pattern Recognition. 2005: 886-893.

[13] Lowe D. Distinctive image features from scale-invariant keypoints. International Journal of Computer Vision. 2004; 60: 91-110.

[14] Ngoc-Son Vu, Alice Caplier. Face Recognition with Patterns of Oriented Edge Magnitudes. In: K. Daniilidis, P Maragos, N Paragios. Editors. ECCV. 2010: 313-326.

[15] PHUNG Van Doanh. Reconnaissance de visages en utilisant le descripteur POEM pattern of oriented Edge Magnitudes, Stage. Institue de la Francophonie pour l'informatique. 2010. Superviseurs: Alice CAPLIER et Ngoc Son VU.

[16] Phillips PJ, Moon H, Rizvi SA, Rauss PJ. The FERET evaluation methodology for face recognition algorithms. IEEE Transactions on Pattern Analysis and Machine Intelligence. 2000; 22(10) : 10901104. 